\title{
Randomized study of digital single-operator cholangioscope compared to fiberoptic single-operator cholangioscope in a novel cholangioscopy bench model
}

\section{(이 $\odot \ominus$}

\author{
Authors
}

Raj J. Shah ${ }^{1}$, Horst Neuhaus ${ }^{2}$, Mansour Parsi ${ }^{3}$, D. Nageshwar Reddy ${ }^{4}$, Douglas K. Pleskow ${ }^{5}$

Institutions

1 Gastroenterology, University of Colorado, Aurora, Colorado, United States

2 Gastroenterology, Evangelisches Krankenhaus, Dusseldorf, Dusseldorf, Germany

3 Gastroenterology, Cleveland Clinic Foundation, Cleveland, United States

4 Gastroenterology, Asian Institute of Gastroenterology, Hyderabad, India

5 Gastroenterology, Beth Israel Deaconess Medical Center, Boston, United States

submitted 22.7.2017

accepted after revision 27.12.2017

Bibliography

DOI https://doi.org/10.1055/a-0584-6458 |

Endoscopy International Open 2018; 06: E851-E856

(c) Georg Thieme Verlag KG Stuttgart · New York

ISSN 2364-3722

Corresponding author

Raj J. Shah, MD, FASGE, AGAF, 1635 Aurora Ct. Mail Stop

F735, AIP 2.031, Aurora, CO 80045

Fax: +1-720-848-2749

raj.shah@ucdenver.edu

\section{ABSTRACT}

Background and study aims Cholangiopancreatoscopy is utilized for diagnosis and therapy of pancreaticobiliary disorders. a fully-disposable, digital, single-operator cholan- gioscope (DSOC) was developed with high image resolution and wide field-of-view. This bench study compared the new DSOC to the previous semi-disposable, fiber-optic cholangioscope (FSOC) prior to the clinical availability of the DSOC system.

Methods Five experts performed one practice run followed by randomized runs comparing DSOC to FSOC in a biliary tract model consisting of three fixed left-intrahepatic tracts (LIHD), and variable common bile duct (CBD) and right-intrahepatic tracts (RIHD) with seven total lesions in multiple configurations. Timed runs aimed to visualize and target each lesion using miniature biopsy forceps. Definitions: visual success, visualizing targets; targeting success, touching target with forceps; complete run, touching seven targets within 20 minutes. Image quality, ease-of-use, and time to completion were recorded.

Results Thirty-seven evaluable runs (20 DSOC, 17 FSOC) were completed. DSOC was superior to FSOC in Visual (99\% vs. $67 \%, P<0.001)$ and targeting success (6.6 vs. 4.5 , $P=0.009)$, proportion of complete runs ( $13 / 20$ vs. $0 / 17, P<$ $0.001)$ and time of run $(10.1 \mathrm{~min}$ vs. $15.4 \mathrm{~min}, P<0.001)$. For fixed LIHD, DSOC achieved higher targeting success compared to FSOC (2.6 vs. $1.1, P<0.001)$ with no difference in RIHD and CBD targets (4.0 vs. 3.4, $P=0.39$ ). Investigators reported superior image quality and ease-of-use with DSOC.

Conclusions In this model, DSOC performed superiorly to FSOC in image quality, visualization, and maneuverability. The model could potentially be utilized for training endoscopists less experienced with cholangiopancreatoscopy.

\section{Introduction}

Peroral cholangiopancreatoscopy (POCP) is used to evaluate indeterminate biliary strictures, intraductal papillary mucinous neoplasia, and treat difficult biliary or pancreatic stones [1]. POCP provides direct visualization of abnormalities not readily identified by radiography; targets stones for intraductal lithotripsy and lesions to biopsy, assessing the extent of tumor for surgical resection; and has emerging applications such as extracting inwardly migrated pancreatic or biliary stents and guiding tumor ablative therapies [2-4].

While use of endoscope-based "mother-daughter" cholangioscopy systems was first reported more than four decades ago, the fiber-optic, single-operator, semi-disposable cholangioscope system (FSOC; Legacy SpyGlass, Boston Scientific Corporation, Marlborough, Massachusetts, United States) has re- 


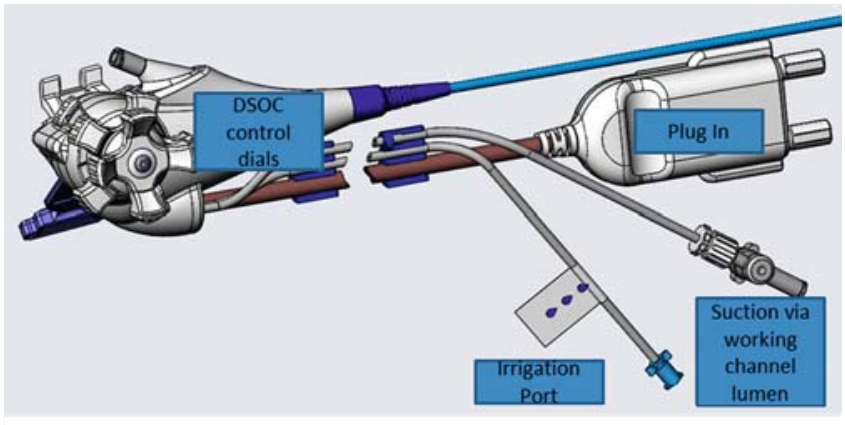

- Fig. 1 SpyGlass Direct Visualization System with SpyScope DS.

sulted in wider utilization of POCP over the last decade. The FSOC was the first commercialized system to integrate optical bundles into a catheter-based system rather than an endoscope-based system [3,4]. Several series describe use of FSOC for evaluation of indeterminate biliary strictures and lesions [5-9]. In a prospective, multi-enter, international clinical registry of 297 patients requiring evaluation of bile duct disease or biliary stone therapy, adequate tissue for histological examination was collected in $88 \%$ of patients who underwent biopsy. Procedure success was achieved in $92 \%$ of patients with stones and the overall incidence of procedure-related adverse events was $7.5 \%$ for diagnostic cases and $6.1 \%$ for stone therapy cases. Importantly, in $64 \%$ of patients, addition of FSOC to endoscopic retrograde cholangiopancreatography led to changes in clinical management [10]. In difficult biliary stone cases, FSOC-guided intraductal lithotripsy has shown complete stone clearance rates of $73 \%$ to $100 \%[11-14]$.

Nearly a decade of clinical experience suggested a need to improve the optical resolution, tip characteristics, accessory introduction, and tip articulation of the FSOC [15-20]. A digital and fully disposable single-operator cholangioscopy system (DSOC; SpyGlass DS, Boston Scientific Corporation, Marlborough, Massachusetts, United States) was developed. The goal of this bench study was to compare FSOC with DSOC in terms of image quality, ease of use, and ability to visualize and target fixed and variable markers utilizing miniature biopsy forceps (MBF, Spybite, Boston Scientific Corporation, Marlborough, Massachusetts, United States).

\section{Materials and methods}

\section{Digital single operator cholangioscopy system}

The DSOC has a 10.8 French access and delivery catheter with a shorter working length for potential use with short wire technology, two dedicated $0.6-\mathrm{mm}$ irrigation channels, and fourway tip deflection. Removal of the fiber-optic cable provided a potential opportunity for additional enhancements. This included a slightly larger working channel diameter through part of its course (maximal diameter $1.3 \mathrm{~mm}$ but tapers to $1.2 \mathrm{~mm}$ ) that may improve the ability to pass accessories, increased maneuverability of the catheter tip, increased field-of-view (120 degrees), and a simplified set-up ( $\bullet$ Fig. 1$)$. Comparative specifications of FSOC with DSOC are detailed in $\triangleright$ Table 1.

\section{Development of the cholangioscopy biliary tract model}

The watertight urethane rubber biliary tract bench model was designed by Pulse Research and Development (Southampton, Pennsylvania, United States) with input from the authors. The model features a fixed section representing a bifurcation $10.5 \mathrm{~cm}$ from the ampulla with a transition to a trifurcation intended to represent acutely angulated left intrahepatic ducts (LIHD). The three LIHD lumina were each $5 \mathrm{~mm}$ in diameter and $35 \mathrm{~mm}$ in length with a visual target at the distal segment designed to represent a $0^{\circ}, 15^{\circ}$, and $30^{\circ}$ angle from the takeoff of the trifurcation. The model also featured two variable inserts representing the common bile duct (CBD) and right intrahepatic (RIHD) tracts. The first insert was approximately $9 \mathrm{~cm}$ long and varied in diameter from $6.5 \mathrm{~mm}$ (RIHD) to $20 \mathrm{~mm}$ (CBD). The pseudopapilla served as the entry of this insert and was $3.8 \mathrm{~mm}$ in diameter. This segment contained two raised lesions (e. g. target areas) of a contrasting color in variable locations. The second insert was approximately $3 \mathrm{~cm}$ long and also contained two colored lesions in variable locations. A total of

- Table 1 FSOC and DSOC specification changes.

\begin{tabular}{|l|l|l|}
\hline \multicolumn{1}{|l|}{ Component } & FSOC & DSOC \\
\hline SpyGlass optical probe & & $120^{\circ}$ \\
\hline Field-of-view & $70^{\circ}$ & Fully-disposable, single-use \\
\hline Disposability & Semi-disposable, up to 10 times reuse & Digital with $4 \times$ greater resolution \\
\hline Camera & Fiber-optic & Digital sensor embedded at the distal tip of the SpyScope \\
\hline Imager & Fiber-optic probe independent of SpyScope & \\
\hline SpyScope access and delivery catheter & & $214 \mathrm{~cm}$ \\
\hline Working length & $220 \mathrm{~cm}$ & 10.8 French \\
\hline Outer diameter & 10 French & $1.3 \mathrm{~mm}$ \\
\hline Working channel diameter & $1.2 \mathrm{~mm}$ & \\
\hline
\end{tabular}




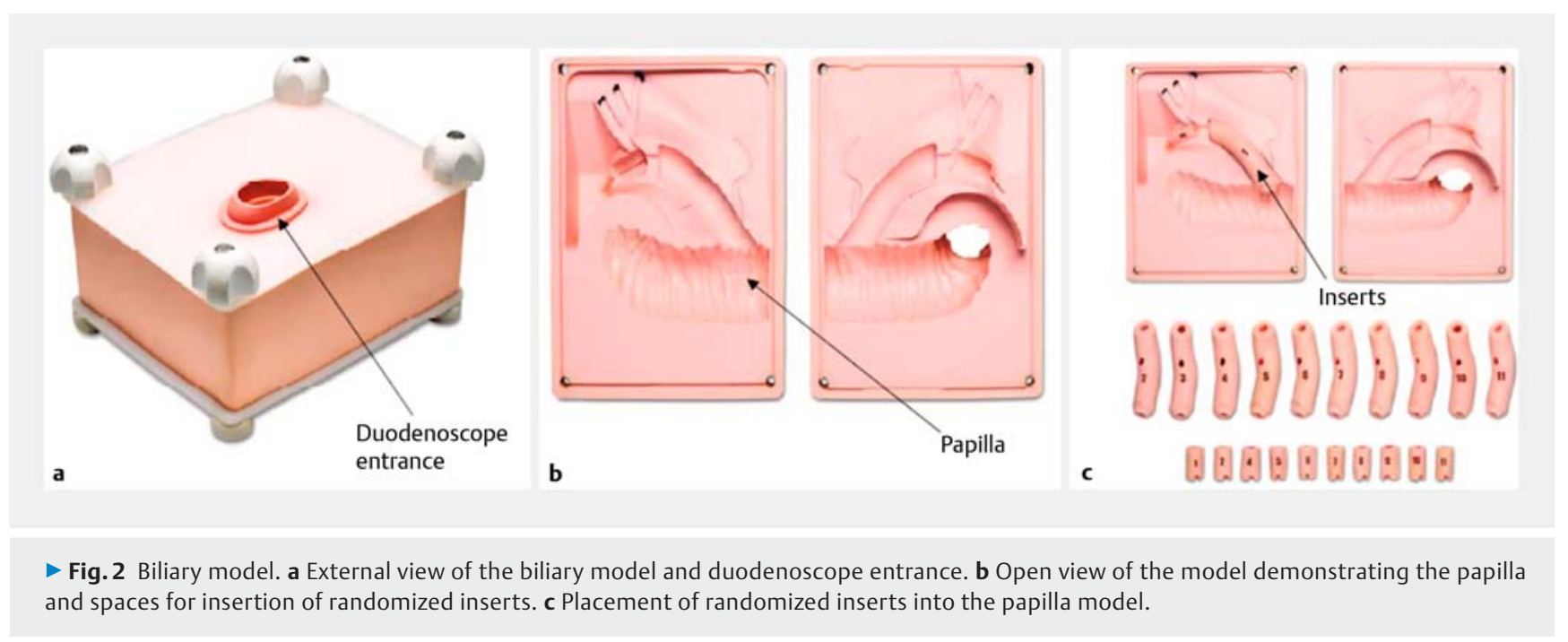

seven lesions were placed inside the LIHD, RIHD, and CBD of the bench model. There were 60 different configurations in total. The model also featured a cystic duct and pancreatic duct which served as visual references only and did not need to be cannulated. Fig. 2 contains an image of the cholangioscopy biliary tract model.

\section{Description of timed trials}

Five investigators from the United States, Asia, and Europe participated in the study. All investigators were experts in the field of cholangioscopy and had utilized FSOC clinically but had only ex-vivo or porcine model experience with the DSOC system prior to initiation of this trial. Immediately prior to the timed trials, each investigator completed a single practice run with both the FSOC and DSOC systems, followed by sequential timed runs randomized to the two systems. Randomization to choose FSOC or DSOC was based on a blinded pull from an allotment of numbers assigned to one of two groups equally represented in the pool (overall 1:1). The goal of each run was to visualize the four variable colored targets and simulate biopsy by touching the target with the MBF which was passed through the system's working channel. In the fixed portion of the model (e.g. LIHD) the goal was to enter and visualize each of the three lumina with terminal targets. A technologist was present during testing to time the run using a stopwatch and document the results. The technologist also changed out the model configurations between runs to provide variations in location of lesions in the model.

The following outcomes were collected to document performance of the devices during testing: Visual success was defined as the ability to visualize the targets. Targeting success was defined as the ability to touch the targets with the MBF. Complete run was defined as identification and targeting of all seven targets within 25 minutes. Total time of run was the duration between entering and exiting the pseudopapilla. A maximum time of 25 minutes was allotted for each run. Each outcome was analyzed by type of target (fixed or variable). Additionally, after completion of all runs, investigators completed a single assessment of image quality and ease of use for FSOC and DSOC using a visual analog scale (VAS) of zero to 10, where zero was unacceptably poor and 10 was excellent.

Data were entered into a database for analysis. All analyses were generated using SAS software version 9.4. (SAS Institute Inc., Cary, North Carolina, United States). Visual success was analyzed using a Fisher's Exact test. Targeting success was analyzed using a negative binomial model between the groups. Time of run and VAS were analyzed using a $t$-test. Time to completion was analyzed using a $t$-test and a negative binomial model was used to test visual and targeting success between the groups.

\section{Results}

A total of 39 runs were completed by five investigators prior to clinical availability of the Spy DS system in February 2015. Data from two of the runs were excluded due to inadequate randomization, resulting in 37 evaluable runs used for analysis (17 FSOC, 20 DSOC; 259 total targets). - Table 2 contains the summary of all results.

Visual success was superior with DSOC compared with FSOC (99\% versus $67 \%, P<0.001)$. As noted in $>$ Fig. 3 , the difference in visual success was more striking with the fixed, acutely angled "LIHD" targets which were correctly identified in $37 \%$ of FSOC runs compared with $98 \%$ of the DSOC runs $(P<0.001)$. The ability to successfully visualize and target (targeting success per run) was superior in the DSOC compared with FSOC groups (6.6 of 7 versus 4.5 of $7, P=0.009$ ). With regard to targeting success, the DSOC group had statistically higher success with fixed targets $(2.6$ versus $1.1, P<0.001)$ but not variable targets $(P=0.449)$. The proportion of complete runs was statistically higher in the DSOC group (13/20, 65\%) compared to the FSOC group $(P<0.001)$.

The time of run was faster with DSOC (average $10.1 \mathrm{~min}$ utes) compared to the FSOC system (average of 15.4 minutes) $(P<0.001)$. In general, the time it took to complete a run improved over time reflecting a learning curve on the part of the 
- Table 2 Time trial outcomes.

\begin{tabular}{|c|c|c|c|}
\hline & $\begin{array}{l}\text { FSOC } \\
17 \text { total trials } \\
119 \text { total targets }\end{array}$ & $\begin{array}{l}\text { DSOC } \\
20 \text { total trials } \\
140 \text { total targets }\end{array}$ & $P$ value \\
\hline Visual success (\%) & $67 \%$ (80 of 119$)$ & $99 \%$ (139 of 140$)$ & $<0.001$ \\
\hline - Variable targets & $90 \%$ (61 of 68$)$ & $100 \%(80$ of 80$)$ & $<0.001$ \\
\hline - Fixed targets & $37 \%(19$ of 51$)$ & $98 \%(59$ of 60$)$ & $<0.001$ \\
\hline $\begin{array}{l}\text { Targeting success per run } \\
\text { Mean (SD) (Min, Max) }\end{array}$ & $\begin{array}{l}4.5(0.7) \\
(3.0,5.0)\end{array}$ & $\begin{array}{l}6.6(0.7) \\
(5.0,7.0)\end{array}$ & 0.009 \\
\hline Variable targets & $\begin{array}{l}3.5(0.7) \\
(2.0,4.0)\end{array}$ & $\begin{array}{l}3.9(0.2) \\
(3.0,4.0)\end{array}$ & 0.449 \\
\hline Fixed targets & $\begin{array}{l}1.1(0.2) \\
(1.0,2.0)\end{array}$ & $\begin{array}{l}2.6(0.7) \\
(1.0,3.0)\end{array}$ & $<0.001$ \\
\hline Complete runs (\%) & $0 \%(0$ of 17$)$ & $65 \%(13$ of 20$)$ & $<0.001$ \\
\hline $\begin{array}{l}\text { Time of run } \\
\text { Mean (SD) (min, max) }\end{array}$ & $\begin{array}{l}15.4(4.0) \\
(7.6,21.6)\end{array}$ & $\begin{array}{l}10.1(3.7) \\
(4.1,17.0)\end{array}$ & $<0.001$ \\
\hline $\begin{array}{l}\text { Image quality (VAS) } \\
\text { Mean (SD) (Min, Max) }\end{array}$ & $\begin{array}{l}4.3(1.0) \\
(3.0,5.0)\end{array}$ & $\begin{array}{l}8.3(0.5) \\
(8.0,9.0)\end{array}$ & $<0.001$ \\
\hline $\begin{array}{l}\text { Ease of use (VAS) } \\
\text { Mean (SD) (Min, Max) }\end{array}$ & $\begin{array}{l}4.8(1.0) \\
(4.0,6.0)\end{array}$ & $\begin{array}{l}8.5(0.6) \\
(8.0,9.0)\end{array}$ & $<0.001$ \\
\hline
\end{tabular}

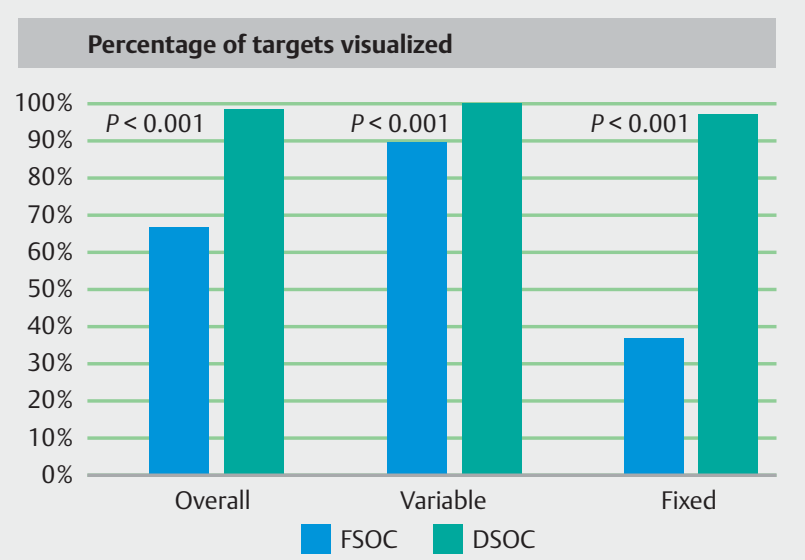

Fig. 3 Visual success.

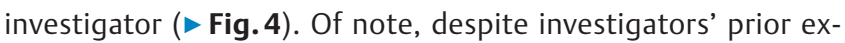
tensive experience with FSOC, both the initial and final time of run was faster with DSOC. Further, the slope of the learning curve was steeper with DSOC.

Each investigator preferred the image quality, as well as the ease of use, with DSOC compared to FSOC ( $>$ Fig.5). On the VAS, average image quality was rated 4.3 with FSOC compared to 8.3 with DSOC $(P<0.001)$. Similarly, ease of use was rated 4.8 with FSOC versus 8.5 with DSOC $(P<0.001)$.

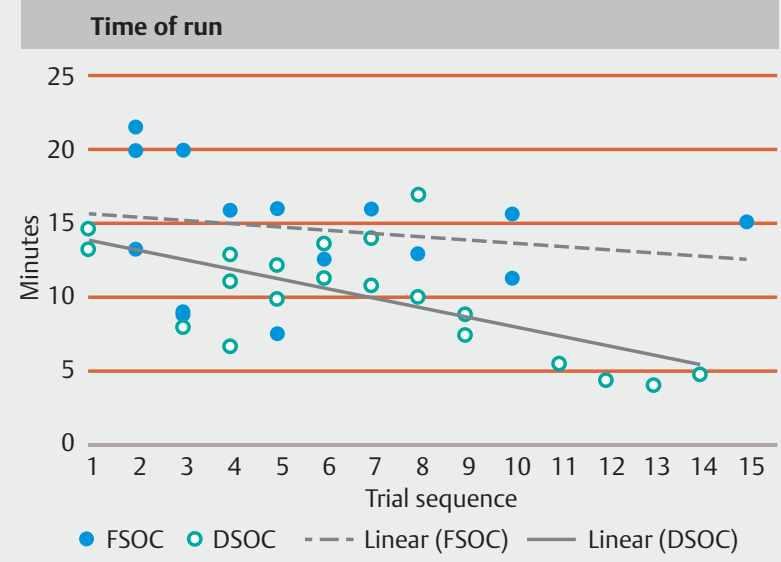

Fig. 4 Time of run learning curve.

\section{Discussion}

Redesign of the fiberoptic device included a tapered insertion tip, digital and higher resolution image, an increased field-ofview from $70^{\circ}$ to $120^{\circ}$, and an increase in working channel diameter through most of its length, which improved upon several of the shortcomings seen with the first-generation SOC system.

Frequently, perceived advances in medical device technology that are often more expensive than their predecessors are incorporated into practice without preclinical bench data to de- 


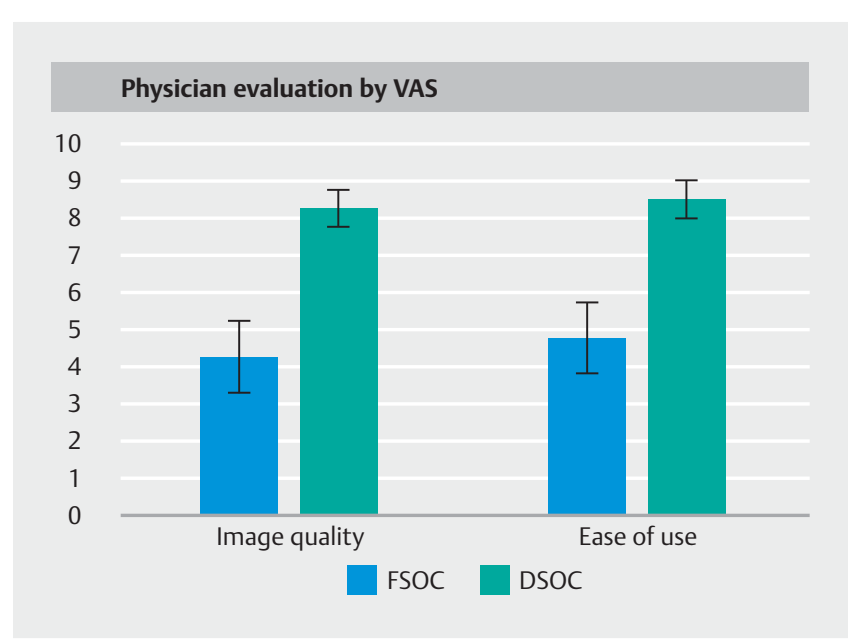

- Fig. 5 Physician evaluation of devices following all runs.

monstrate superiority. Endoscopic devices are no exception and though limited retrospective clinical data using DSOC do exist, objective comparative studies are lacking [21,22]. Results from our bench model study which was performed prior to the clinical availability of the DSOC system suggest that these theoretical improvements, though unblinded, have been objectively realized with significantly higher rates of visual and targeting success and reduced total times with DSOC. Of note, no investigator was able to achieve a complete run ( 7 of 7 targets identified and simulated biopsies completed) with the FSOC in the allotted time primarily due to the limited tip articulation of the fiber-optic system. A difference in targeting of the CBD lesions was not seen between the two arms likely due to minimal need for tip angulation. When utilizing the DSOC, the physicians achieved complete runs in $65 \%$ of cases. Clinical implications for this finding may be an enhanced ability to enter tortuous intrahepatic ducts, cystic duct insertion points, and traversing and visualizing the often-angulated genu during pancreatoscopy.

Though efficiency is an ever-increasing requirement for endoscopists, visualizing pathology and targets with confidence by an improved optical image is essential. To further enhance our ability and interest in utilizing new technology, an improvement in device set-up is apparent. The device has automatic illumination, white balance and focus with a "plug-andplay" configuration given the absence of a separate optical bundle as in the FSOC system. Clinically, this may result in shorter procedure times with potential reductions in anesthesia time and fluoroscopy use. Further, the DSOC device is fully disposable which eliminates the need for reprocessing of the optical bundle that was required with FSOC [4].

Although each endoscopist had extensive experience with FSOC and limited to no experience with DSOC, each individual adapted to use of DSOC with quicker times to completion and more complete trials, demonstrating the impact of the improved image quality and maneuverability.
The ex-vivo biliary model utilized in this study had visual targets and angulations that may be seen during examination of the extrahepatic ducts and tortuous intrahepatic ducts, respectively. Unique aspects of this ex-vivo biliary model study were the blinding of endoscopists to the location of targets in the extrahepatic exchangeable segment and ability to standardize and quantify visual targets for biopsy, which permitted a direct comparison of the two devices.

Several limitations of this study warrant discussion. First, the investigators who participated in the study were experienced users of the FSOC system and the ability to adapt to potentially improved image quality and maneuverability with DSOC was seamless. This may not translate to a simple transition for less experienced users. The endoscopists were not blinded to type of catheter used as the reusable and movable optical probe in the $\mathrm{FSOC}$ is a visible distinguishing feature. Further, their knowledge and, in some cases, input for development of the new device could have introduced bias in their utilization of it. The investigators did not have a set minimum or maximum time limit to identify each individual target, which may have influenced the time of run but each investigator was aware of the total time restriction. It is nevertheless encouraging that the results of the objective measures of time and successful visualization correlate with the physicians' purely subjective perceptions of their experience according to the VAS. Use of the bench model, while convenient and anatomically simulating acute intrahepatic angulations, made it difficult to collect actual biopsy samples. Therefore, the surrogate measure of touching the targets with forceps was as close to simulating a biopsy as possible but would not be able to assess tissue adequacy. Extrahepatic portions of the model were interchangeable so investigators were not aware of the location of lesions in this area with each pass through. The bench model was not able to simulate some real-world clinical situations such as use of irrigation and suction to clear debris and mucus from the visual field or passage of accessories such as biopsy forceps through the two devices' working channels or wire exchanges for advancement of the SOC system.

\section{Conclusion}

In conclusion, in this ex-vivo biliary bench model, the digital single-operator cholangioscopy system demonstrated superior performance when compared with the fiber-optic single-operator system. The DSOC has improved digital image capability and maneuverability. Further, and perhaps most importantly as continued expanded use of intraductal endoscopy is realized, this novel bench biliary model could be utilized in training endoscopists that are less experienced with cholangiopancreatoscopy.

Further, whether the simpler setup and ease of use will permit wider adoption by high-volume ERCP users new to cholangioscopy remains to be seen. 


\section{Acknowledgements}

The authors wish to dedicate this manuscript to the memory of Professor Yang K. Chen, MD, FASGE, AGAF, FACG, who pioneered the widespread clinical application of per oral cholangiopancreatoscopy.

\section{Competing interests}

Drs. Shah, Neuhaus, Parsi, Reddy, and Pleskow serve on the medical advisory board and are consultants for Boston Scientific, Inc.

\section{References}

[1] Chen YK. Preclinical characterization of the Spyglass peroral cholangiopancreatoscopy system for direct access, visualization, and biopsy. Gastrointest Endosc 2007; 65: 303-311

[2] Ramchandani M, Reddy DN, Lakhtakia S et al. Per oral cholangiopancreatoscopy in pancreatico biliary diseases - Expert consensus statements. World J Gastroenterol 2015; 21: 4722-4734

[3] Arvanitakis M, Devière ]. Innovations. Endoscopy 2008; 40: 152 - 155

[4] ASGE Technology Committee. Shah RJ, Adler DG, Conway JD et al. Cholangiopancreatoscopy. Gastrointest Endosc 2008; 68: 411-421

[5] Ramchandani M, Reddy DN, Gupta R et al. Role of single-operator peroral cholangioscopy in the diagnosis of indeterminate biliary lesions: a single-center, prospective study. Gastrointest Endosc 2011; 74: $511-519$

[6] Navaneethan U, Hasan MK, Lourdusamy V et al. Single-operator cholangioscopy and targeted biopsies in the diagnosis of indeterminate biliary strictures: a systematic review. Gastrointest Endosc 2015; 82: $608-614$

[7] Draganov PV, Chauhan S, Wagh MS et al. Diagnostic accuracy of conventional and cholangioscopy-guided sampling of indeterminate biliary lesions at the time of ERCP: a prospective, long-term follow-up study. Gastrointest Endosc 2012; 75: 347 - 353

[8] Chen YK, Pleskow DK. SpyGlass single-operator peroral cholangiopancreatoscopy system for the diagnosis and therapy of bile-duct disorders: a clinical feasibility study (with video). Gastrointest Endosc 2007; 65: $832-841$
[9] Draganov PV, Lin T, Chauhan S et al. Prospective evaluation of the clinical utility of ERCP-guided cholangiopancreatoscopy with a new direct visualization system. Gastrointest Endosc 2011; 73: 971 - 979

[10] Chen YK, Parsi MA, Binmoeller KF et al. Single-operator cholangioscopy in patients requiring evaluation of bile duct disease or therapy of biliary stones (with videos). Gastrointest Endosc 2011; 74: 805-814

[11] Seelhoff A, Schumacher B, Neuhaus H. Single operator peroral cholangioscopic guided therapy of bile duct stones. J Hepatobiliary Pancreat Sci 2011; 18: $346-349$

[12] Maydeo A, Kwek BE, Bhandari S et al. Single operator cholangioscopyguided laser lithotripsy in patients with difficult biliary and pancreatic ductal stones (with videos). Gastrointest Endosc 2011; 74: 1308 1314

[13] Kalaitzakis E, Webster G], Oppong KW et al. Diagnostic and therapeutic utility of single-operator peroral cholangioscopy for indeterminate biliary lesions and bile duct stones. Eur J Gastroenterol Hepatol 2012; 24: 656-664

[14] Aljebreen AM, Alharbi OR, Azzam N et al. Efficacy of spyglass-guided electrohydraulic lithotripsy in difficult bile duct stones. Saudi J Gastroenterol 2014; 20: 366-370

[15] Kalaitzakis E, Webster G], Oppong KW et al. Diagnostic and therapeutic utility of single-operator peroral cholangioscopy for indeterminate biliary lesions and bile duct stones. Eur J Gastroenterol Hepatol 2012; 24: 656-664

[16] Nguyen NQ. Getting most out of SpyGlass cholangio-pancreatoscopy: how and when? J Gastroenterol Hepatol 2012; 27: 1263 - 1265

[17] Kawakubo K, Isayama H, Sasahira N et al. Clinical utility of single-operator cholangiopancreatoscopy using a SpyGlass probe through an endoscopic retrograde cholangiopancreatography catheter. J Gastroenterol Hepatol 2012; 27: 1371 -1376

[18] Kahaleh M. Spyglass direct visualization system. Clin Endosc 2012; 45: $316-318$

[19] Draganov P. The SpyGlass $®$ Direct Visualization System for cholangioscopy. Gastroenterol Hepatol (NY) 2008; 4: 469-470

[20] Moon JH, Terheggen G, Choi H] et al. Peroral cholangioscopy: diagnostic and therapeutic applications. Gastroenterology 2013; 144: $276-282$

[21] Shah RJ, Raijman I, Brauer B et al. Performance of a fully disposable, digital, single-operator cholangiopancreatoscope. Endoscopy 2017; 49: $651-658$

[22] Navaneethan U, Hasan MK, Kommaraju K et al. Digital, single-operator cholangiopancreatoscopy in the diagnosis and management of pancreatobiliary disorders: a multicenter clinical experience (with video). Gastrointest Endosc 2016; 84: 649-655 